\title{
Effects of VEGF on Bone Cells' Metabolism and Activity
}

\author{
Yang $\mathrm{Y}^{1^{*}}$, Chai $\mathrm{L}^{2}$, Liao $\mathrm{C}^{1}$ and $\mathrm{Qu} \mathrm{JY}^{3}$
}

${ }^{1}$ Faculty of Dentistry, The University of Hong Kong, Hong Kong SAR, China

${ }^{2}$ School of Dentistry, University of Queensland, Brisbane, Australia

${ }^{3}$ Biomedical Engineering Program, Department of Electronic and Computer Engineering, Hong Kong University of Science and Technology, Hong Kong SAR, China

"Corresponding author: Dr. Yang Yan-Qi BDS, MOrthRCS (Edin), PhD, FDS RCS (Edin), Assistant Professor, Faculty of Dentistry, The University of Hong Kong, The Prince Philip Dental Hospital, 34 Hospital Road, Hong Kong, Tel: (852) 2859 0252; Fax: (852) 2559 3803; E-mail: yangyanq@hku.hk

Rec date: Apr 01, 2014, Acc date: Jul 14, 2014, Pub date: Jul 16, 2014

Copyright: (C) 2014 Yang Y, et al. This is an open-access article distributed under the terms of the Creative Commons Attribution License, which permits unrestricted use, distribution, and reproduction in any medium, provided the original author and source are credited

\begin{abstract}
The treatments of bone loss have been extended to the molecular level and previous research has found that vascular endothelia growth factor (VEGF) can enhance the vascular network building around bone injury to aid healing progress. In this manuscript, we discussed the effects of VEGF on bone cells' metabolism and activity. VEGF could enhance osteogenesis by inducing neovascularization and direct effects on bone cells via regulation on genes osteoprotegerin (OPG)/the receptor activator of nuclear factor kappa beta ligand (RANKL), alkaline phosphatase (ALP), osteopontin (OPN) and osteocalcin (OCN), without causing abnormal cell metabolism, which may shed lights on new clinical treatments of bone loss.
\end{abstract}

Keywords Vascular endothelia growth factor; Bone remodeling; Cell metabolism

\section{Introduction}

Bone loss, caused by diseases, traumas and surgical procedures, is a problem resulting in dental or craniofacial defects and even leading to ever-increasing social impacts. Traditional approaches of the bone repair and regeneration include distraction osteogenesis, orthognathic surgery, bone grafting, et al., which were limited by bone graft resorption, donor site morbidity, antigenicity potential and disease transmission [1]. Hence, there is an urgent need to explore new improvement in bone induction and regeneration [2].

Blood supply is the essential factor of bone regeneration. Angiogenesis, the ingrowth of new capillary blood vessels, is a critical component of skeletal development, which is closely correlated to the success of bone regeneration [3,4]. Hence, a growth factor which can enhance both angiogenesis and osteogenesis is ideal for the search as the addition of such a factor may be a method to improve the outcomes of bone formation [5].

Vascular endothelia growth factor (VEGF), a key regulator of angiogenesis and osteogenesis during bone regeneration, has been found to be highly expressed in vascularized tissues [6]. Neonatal mice with conditional knockouts of VEGF in the growth plates had great problems in bone formation, which confirmed the role of VEGF in bone development [7].

In addition to enhancing bone formation by increasing vascularization, Does VEGF have direct effect on bone cells which contributes to osteogenesis? In this manuscript, we would like to review and discuss the roles of VEGF involved in the following aspects: initial removal of 'old' bone; bone formation; dynamic balance of bone remodeling; and effects on cell metabolism.

\section{Initial Removal of Old Bone}

The initial step of bone remodeling is removal of 'old' bone to provide places for new bone formation [8]. Osteoblasts play an essential role in osteogenesis, and are also responsible for differentiation and maturation of osteoclasts $[9,10]$. It was clear that osteoprotegerin (OPG) and the receptor activator of nuclear factor kappa beta ligand (RANKL) are two important regulatory factors in bone resoprtion, which can be expressed by osteoblasts, and also involve in regulating activation and function of osteoclasts [11,12].

In order to investigate the effects of VEGF on bone remodeling, MC3T3-E1, one of the mouse osteoblast precursor cell lines, was incubated with VEGF [8]. Cells were collected at different time points $(24,48$ and $72 \mathrm{hrs})$ respectively and total RNA was isolated immediately. Real-time polymerase chain reaction (qPCR) was carried out to quantify the mRNA expression of OPG and RANKL [8].

\begin{tabular}{|l|l|l|l|}
\hline & 24 hrs & 48 hrs & 72 hrs \\
\hline OPG & $\downarrow^{* * *}$ & NS & $\uparrow^{* * *}$ \\
\hline RANKL & NS & NS & NS \\
\hline OPG/RANKL & $\downarrow^{* * *}$ & NS & $\uparrow^{* * *}$ \\
\hline \multirow{2}{|l}{} \\
\hline
\end{tabular}

Table 1: Effects of VEGF on expression of OPG and RANKL in MC3T3-E1 bone cells [8]

In this study [8], it was shown that after VEGF induction, the mRNA expression of OPG significantly dropped $7 \%$ compared to control at the time point of $24 \mathrm{hrs}(\mathrm{p}<0.001)$, while showed no difference from control at $48 \mathrm{hr}(\mathrm{p}>0.05)$. But at $72 \mathrm{hrs}$, OPG increased dramatically by $133 \%$ compared to control $(\mathrm{p}<0.001)$ [8]. There was no difference in the expression of RANKL between test and control groups at any time point. Thus the ratio OPG/RANKL declined at first and then rose later [8]. The data was summarized in Table 1. 
There are several important steps in bone resorption: recruitment of osteoclasts, differentiation of osteoclasts, dissolution of bone minerals, and degradation of organic matrix [12]. Osteoblasts express RANKL as a membrane associated factor, and osteoclast precursors express RANK, a receptor for RANKL [13]. By direct cell-to-cell contact, osteoclast precursors recognize RANKL and differentiate into osteoclasts. Therefore, osteoblasts play an essential role in osteoclasts differentiation. OPG, a decoy receptor for RANKL secreted by osteoblasts, can suppress the links between RANKL and RANK and further block their functions in osteoclastogenesis and bone resorption [14]. The balanced expression ratio of OPG/RANKL was suggested to be crucial for bone remodeling [15].

It was shown in Table 1 that the mRNA expression of OPG decreased significantly while RANKL kept unchanged at the beginning ( $24 \mathrm{hrs}$ ), leading to the decrease of OPG/RANKL expression ratio in MC3T3-E1 at the early phase by VEGF induction. The reduced OPG/ RANKL ratio suggests bone resorption was enhanced, conducive to the removal the 'old' bone as an essential trigger of bone remodeling. These findings indicated that VEGF may promote bone resoprtion at the early time of bone remodeling, neovascularization and revitalization of the dead bone.

\section{Bone Formation}

Following the removal of 'old' bone, osteoblasts differentiated from osteoprogenitor cells arise at resorption site and participate in bone formation [9]. MC3T3-E1 which comes from osteoblast precursor cell line can differentiate along the osteoblast pathway [16]. In order to investigate the effect of VEGF mediating bone formation, using the same in vitro model of MC3T3-E1 cell cultured with VEGF [8], Alkaline phosphatase (ALP), osteopontin (OPN) and osteocalcin $(\mathrm{OCN})$ were chosen as the markers [8], all of which are common bone formation factors [17,18]. ALP is known to be responsible for osteoblastic differentiation [17]. OPN is a major non-collagenous bone matrix protein secreted into the mineralizing extracellular matrix by osteoblasts during bone development [18]. OCN is closely related to calcification [19].

\begin{tabular}{|l|l|l|l|}
\hline & 24 hrs & 48 hrs & 72 hrs \\
\hline ALP & $\uparrow^{* * *}$ & $\downarrow^{* * *}$ & $\downarrow^{*}$ \\
\hline OPN & $\downarrow^{* * *}$ & NS & $\uparrow^{* * *}$ \\
\hline OCN & $\downarrow^{* * *}$ & NS & $\uparrow^{* * *}$ \\
\hline \multirow{2}{*}{ * $(p<0.05) ;{ }^{* * *}(p<0.001) ;$ NS (Not Significant) } \\
\hline
\end{tabular}

Table 2: Effects of VEGF on expression of ALP, OPN and OCN in MC3T3-E1 bone cells $[8,20]$

It was found that the mRNA expression of ALP increased by $73 \%$ after VEGF incubation for $24 \mathrm{hrs}(\mathrm{p}<0.001)$, but reduced by $14 \%$ and $41 \%$ at $48 \mathrm{hrs}(\mathrm{p}<0.001)$ and $72 \mathrm{hrs}(\mathrm{p}<0.05)$ respectively when compared to the control [8]. For the other two factors, OPN expression decreased by $11 \%(\mathrm{p}<0.001)$ and $\mathrm{OCN}$ dropped by $41 \%$ $(\mathrm{p}<0.001)$ at $24 \mathrm{hrs}$; both the expression of OPN and OCN did not show any change at $48 \mathrm{hrs}$; but at $72 \mathrm{hrs}$, OPN increased by $94 \%$ $(\mathrm{p}<0.001)$ and OCN grew by $149 \%(\mathrm{p}<0.001)[8,20]$. The data was summarized in Table 2 . The dramatic upregulation of the three bone formation related markers at different time indicate the enhancement of bone formation by VEGF, and the time-related change is coincide with three markers' chronological function in osteogenesis progress.

\section{Dynamic Balance of Bone Remodeling}

Bone remodeling, the two sub-processes bone formation and resorption, is a lifelong dynamic balance process, determined by the function of osteoblasts and osteoclasts, and precisely mediated by various cytokines during development and maintenance of the skeleton [21]. Any dysregulation in development of osteoclast and osteoblast or the disruption of the balance between the sub-processes will result in bone diseases. For the roles of VEGF on bone cells, we would like to highlight two aspects of the dynamic balance.

For bone resorption, OPG/RANKL ratio degreased at early phase (24 hr) in MC3T3-E1 by VEGF induction, indicating more bone resorption for removal of old bones in initial phase [8]. However, at later phase $(72 \mathrm{hrs})$, the increased OPG/RANKL ratio showed that bone resorption was suppressed at the later stage of bone remodeling [8].

For bone formation, ALP, OPN and OCN were found increasing at different phases of intervention $[8,20]$. The process of osteogenesis consists of cell proliferation, cell differentiation, and mineralization, and ALP, OPN and OCN are related to the different stages of this process: ALP is an early marker, which is known to be responsible for osteoblastic differentiation; while OPN and OCN are regarded as late markers, as OPN is a major non-collagenous bone matrix protein secreted into the mineralizing extracellular matrix, and OCN is also closely related to calcification [17-19]. It was shown that the increase of ALP happened at the early stage (24 hrs) [8], while OPN and OCN had a significant increase at late stage $(72 \mathrm{hrs})[8,20]$, consistent with the different time-serial function of ALP, OPN and OCN, indicating that VEGF play different regulation roles in different bone formation stages.

\section{Effects on Cell Metabolism}

Growth factors, like VEGF, a group of special polypeptides that stimulate cell proliferation, are major growth-regulatory molecules for cells in culture and probably in vivo [22]. So the excitement and attention of growth factors focus on not only the regulation of normal cell growth but also the involvement of growth factor-initiated pathways causing abnormal cell metabolism and even in the etiology of cancer [22].

Nicotinamide adenine dinucleotide (NADH), which can emit strong fluorescence, has been used to probe cell activity for many years [23], as the ratio of free/enzyme-bound NADH differentiated by their fluorescence decay is a good indicator of the cell metabolic state [24,25]. Tryptophan is one kind of amino acids acting as building blocks in protein, the fluorescence intensity of which can convey the information about protein content, protein structure, and changes in the cellular microenvironment [26].

We have built a time-resolved two-photon excitation fluorescence microscopy and spectroscopy system equipped with a multichannel time-correlated single-photon-counting facility [27]. This system provides a unique approach to measure fluorescence signals of NADH and tryptophan in different cellular organelles and the cytoplasm. Importantly, the system detects fluorescence signals in living cells without killing them, thereby permitting real-time observations of cellular endogenous fluorescence with different intervention [28,29]. 
Page 3 of 4

To investigate the effect of VEGF on bone cells' metabolism, MC3T3-E1 was cultured with VEGF. The endogenous fluorescence of $\mathrm{NADH}$ and tryptophan was measured at different culture time points (24,48 and $72 \mathrm{hrs}$ ) with the two-photon autofuorescence microscopy which can measure three types of characteristics of the autofluorescence, i.e. the intensity distribution in 2D area named fluorescence image; the fluorescence spectra; and the fluorescence lifetime. In the measurement of both $\mathrm{NADH}$ and tryptophan fluorescence (Figure 1), regarding the three characteristics, there was no difference between the VEGF treated and control cells. It was suggested that application of the growth factor VEGF does not cause abnormal cell metabolism.
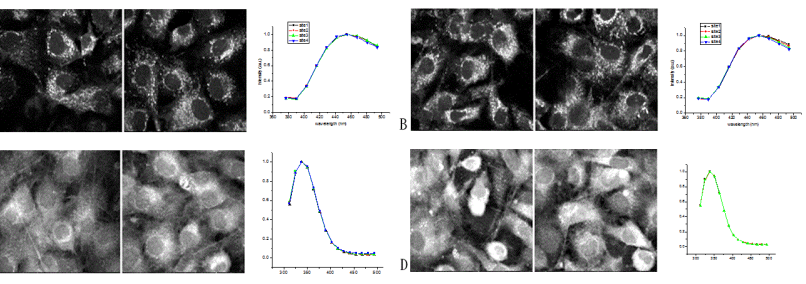

Figure 1: Fluorescence image and spectra. A, NADH fluorescence image and spectra of control cells ( $72 \mathrm{hrs}$ ); B, NADH fluorescence image and spectra of VEGF treated cells (72 hrs); C, Tryptophan fluorescence image and spectra of control cells (72 hrs); D, Tryptophan fluorescence image and spectra of VEGF treated cells (72 hrs). There were no difference between the VEGF treated and control cells, which suggested that application of the growth factor of VEGF does not cause the abnormal cell metabolism

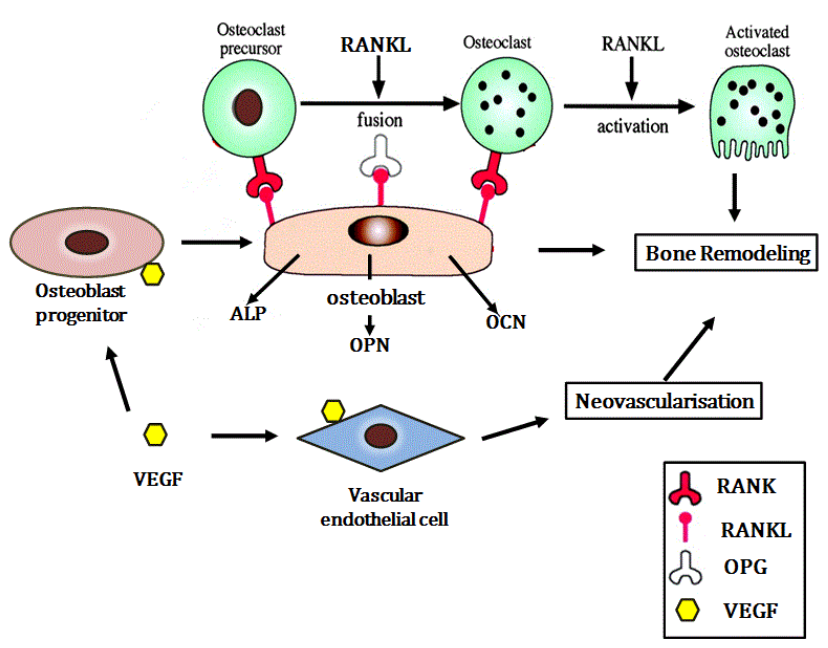

Figure 2: Effects of VEGF on bone remodeling

In conclusion, VEGF could play an important role in osteogenesis enhancement by inducing neovascularization and direct effects on bone cells via regulation on genes ALP, OPN, OCN and OPG/RANKL (Figure 2) without causing abnormal cell metabolism. The pivotal role of VEGF in bone remodeling and healing may shed lights on the new clinical treatment of bone loss.

\section{References}

1. Rabie ABM, Dai J, Xu R (2007) Recombinant AAV-mediated VEGF gene therapy induces mandibular condylar growth. Gene ther 14: 972-980.

2. Dai J, Rabie ABM, Hagg U, Xu R (2004) Alternative gene therapy strategies for the repair of craniofacial bone defects. Curr Gene Ther 4: 469-485.

3. Gerber HP, Ferrara N (2000) Angiogenesis and bone growth. Trends Cardiovasc Med 10: 223-228.

4. Olsen BR, Reginato AM, Wang W (2000) Bone development. Annu Rev Cell Dev Biol 16: 191-220.

5. Marden LJ, Reddi HA, Hollinger JO (1990) Growth and differentiation factors: role in bone induction and potential application in craniofacial surgery. J Craniofac Surg 1: 154-160.

6. Ferrara N, Henzel WJ (1989) Pituitary follicular cells secrete a novel heparin-binding growth factor specific for vascular endothelial cells. Biochem Biophys Res Commun 161: 851-858.

7. Haigh JJ, Gerber HP, Ferrara N, Wagner EF (2000) Conditional inactivation of VEGF-A in areas of collagen2a1 expression results in embryonic lethality in the heterozygous state. Development 127: 1445-1453.

8. Tan YY, Yang YQ, Chai L, Wong RWK, Rabie ABM (2010) Effects of vascular endothelial growth factor (VEGF) on MC3T3-E1. Orthod Craniofac Res 13: 223-228.

9. Suda T, Takahashi N, Martin TJ (1992) Modulation of osteoclast differentiation. Endocr Rev 13: 66-80.

10. Suda T, Udagawa N, Nakamura I, Miyaura C, Takahashi N (1995) Modulation of osteoclast differentiation by local factors. Bone 17: 87S-91S.

11. Yasuda H, Shima N, Nakagawa N, Yamaguchi K, Kinosaki M, et al. (1999) A novel molecular mechanism modulating osteoclast differentiation and function. Bone 25: 109-113.

12. Katagiri T, Takahashi N (2002) Regulatory mechanisms of osteoblast and osteoclast differentiation. Oral Dis 8: 147-159.

13. Feng X (2005) Regulatory roles and molecular signaling of TNF family members in osteoclasts. Gene 350: 1-13.

14. Kanzaki H, Chiba M, Sato A, Miyagawa A, Arai K, et al. (2006) Cyclical tensile force on periodontal ligament cells inhibits osteoclastogenesis through OPG induction. J Dent Res 85: 457-462.

15. Neves JS, Salmon CR, Omar NF, Narvaes EAO, Gomes JR, et al. (2009) Immunolocalization of CSF-1, RANKL and OPG in the enamel-related periodontium of the rat incisor and their implications for alveolar bone remodeling. Arch Oral Biol 54: 651-657.

16. Wong RWK, Rabie ABM (2008) Effect of quercetin on preosteoblasts and bone defects. Open Orthop J 2: 27-32.

17. Christenson RH (1997) Biochemical markers of bone metabolism: an overview. Clin Biochem, 30: 573-593.

18. Jang JH, Kim JH (2005) Improved cellular response of osteoblast cells using recombinant human osteopontin protein produced by Escherichia coli. Biotechnol Lett 27: 1767-1770.

19. Lee AJ, Hodges S, Eastell R (2000) Measurement of osteocalcin. Ann Clin Biochem 37: 432-446.

20. Wenden A, Yang Y, Chai L, Wong RW (2013) Salvia Miltiorrhiza Induces VEGF Expression and Regulates Expression of VEGF Receptors in Osteoblastic Cells. Phytother Res 28: 673-677.

21. Manolagas SC, Jilka RL (1995) Bone marrow, cytokines, and bone remodeling. Emerging insights into the pathophysiology of osteoporosis. New Engl J Med 332: 305-311.

22. Goustin AS, Leof EB, Shipley GD, Moses HL (1986) Growth factors and cancer. Cancer Res 46: 1015-1029.

23. Chance B, Cohen P, Jobsis F, Schoener B (1962) Intracellular oxidationreduction states in vivo. Science 137: 499-508.

24. Salmon JM, Kohen E, Viallet P, Hirschberg JG, Wouters AW, et al. (1982) Microspectrofluorometric approach to the study of free/bound 
Citation: Yang Y, Chai L, Liao C, Qu JY (2014) Effects of VEGF on Bone Cells' Metabolism and Activity. Dentistry 4: 247. doi: 10.4172/2161-1122.1000247

Page 4 of 4

$\mathrm{NAD}(\mathrm{P}) \mathrm{H}$ ratio as metabolic indicator in various cell types. Photochem Photobiol 36: 585-593.

25. Pradhan A, Pal P, Durocher G, Villeneuve L, Balassy A, et al. (1995) Steady state and time-resolved fluorescence properties of metastatic and non-metastatic malignant cells from different species. J Photochem Photobiol B 31: 101-112.

26. Mujat C, Greiner C, Baldwin A, Levitt JM, Tian F, et al. (2008) Endogenous optical biomarkers of normal and human papillomavirus immortalized epithelial cells. Int J Cancer 122: 363-371.
27. Li D, Zheng W, Qu JY (2009) Two-photon autofluorescence microscopy of multicolor excitation. Opt Lett 34: 202-204.

28. Wu Y, Zheng W, Qu JY (2006) Sensing cell metabolism by time-resolved autofluorescence. Opt Lett 31: 3122-3124.

29. Li D, Zheng W, Qu JY (2008) Time-resolved spectroscopic imaging reveals the fundamentals of cellular NADH fluorescence. Opt Lett 33: 2365-2367. 